\title{
Plantas usadas por aves en paisajes cafeteros de Nariño, Colombia
}

\author{
Plant used by birds in coffee landscapes of Nariño, Colombia \\ Yulieth Viviana Castillo R. ${ }^{1}$; Jhon Jairo Calderón L. ${ }^{2}$
}

1 Bióloga. Grupo de investigación en Ecología Evolutiva-Universidad de Nariño, Pasto, Nariño, Colombia, yuliethcastillo@gmail.com.

2 Docente, M.Sc. Ciencias Biológicas. Grupo de investigación en Ecología Evolutiva, Universidad de Nariño, Pasto, Nariño, Colombia, jjcalderl@gmail.com.

Citar: Castillo, Y.; Calderón, J. 2017. Plantas usadas por aves en paisajes cafeteros de Nariño, Colombia. Rev. Cienc. Agr. 34(2): 3 - 18. doi: http://dx.doi.org/10.22267/rcia.173402.68.

Recibido: Agosto 04 de $2016 . \quad$ Aceptado: Agosto 08 de 2017.

\begin{abstract}
RESUMEN
Las plantas son un importante recurso alimenticio para las aves presentes en los paisajes cafeteros, por lo que la heterogeneidad en las diferentes unidades de estos paisajes, garantizan la disponibilidad de alimento. Conocer este tipo de interacciones entre plantas y aves es importante para la conservación de la biodiversidad en paisajes cafeteros. En esta investigación, se presenta la lista inicial de especies de plantas usadas por las aves de paisajes cafeteros en zonas rurales de los municipios de Colón-Génova, La Unión, Arboleda y San Pedro de Cartago en el departamento de Nariño, Colombia. Las observaciones en campo y colectas generales de plantas muestran que 25 especies vegetales son consumidas por aves frugívoras, ocho especies son aprovechadas por aves nectarívoras y nueve especies son aprovechadas por aves insectívoras. Los resultados obtenidos permiten proponer un listado de especies de plantas potenciales como fuente de recursos para las aves, algunas nativas y otras introducidas, que pueden ser útiles para el manejo de paisajes cafeteros en la región a través de la implementación de estrategias de producción y propagación para la restauración.
\end{abstract}

Palabras claves: conservación, biodiversidad florística, avifaunística, restauración. 


\begin{abstract}
Plants are an important food resource for birds in coffee landscapes and heterogeneous coffee plantations guarantee resource availability. Understanding the interactions between birds and plants is important for management and biodiversity conservation in coffee landscapes. In this investigation, we present an initial list of plant species used by birds in coffee landscapes in rural areas of the municipalities of Colón-Génova, La Unión, Arboleda and San Pedro de Cartago (Nariño, Colombia). The results showed that frugivorous birds fed from at least 25 species, nectarivorous birds from eight and insectivorous birds foraged in nine. The results obtained allow us to propose a list of potential plants species (native and introduced), used as a food and foraging resource by birds, which could be useful for coffee landscapes management through the implementation of restoration propagative and reproductive strategies.
\end{abstract}

Keywords: conservation, floristic biodiversity and birdlife, restoration.

\section{INTRODUCCIÓN}

En Colombia, la región cafetera está ubicada entre los 1000 y 2000 msnm, en una franja que cubre varios tipos de ecosistemas a lo largo de la región andina. Esta franja ha sido reconocida de importancia ecológica, con altos valores de diversidad y endemismo (Rappole et al., 2003). En Nariño, los cultivos de café cubren un área de 32.068ha, menos del 1\% de la superficie del departamento y alcanzan alturas hasta $2300 \mathrm{msnm}$, superiores a las tradicionales para el resto del país. Pese a esta baja proporción de cobertura de las plantaciones de café respecto al área del departamento, hay una elevada tasa de pérdida de la cobertura vegetal original y solo quedan remanentes boscosos inmersos en la matriz cafetera de estos paisajes (Giraldo et al., 2009). La transformación de los cultivos tradicionales cafeteros de sombra en monocultivos expuestos directamente al sol o a libre exposición y con ello, los cambios en la cobertura vegetal, ha generado una reducción en los servicios, bienes ecosistémicos y variación en las características estructurales de las comunidades presentes como variación en la cantidad y composición de las especies (Borrero, 1986).

Para contrarrestar esta pérdida e intentar recuperar las funciones y valores originales de estos sistemas, se han generado iniciativas con herramientas de manejo del paisaje, que están orientadas a lograr que los paisajes cafeteros funcionen como ecosistemas amigables con la flora y fauna local, donde el crecimiento socio-económico y la conservación de la biodiversidad y sus procesos biológicos, trabajan conjuntamente. Varias de estas iniciativas proponen no sólo proteger los remanentes de cobertura original, sino conectar e incrementar esta cobertura por medio de estrategias que permitan el manejo y la implementación de sistemas adecuados en el cultivo de café, reconociendo a los cultivos de café bajo un manejo agroforestal como importantes reservorios de diversidad biológica y refugios para las aves de áreas boscosas (Perfecto et al., 1996; Borkhataria et al., 2012).

Considerando la continua disminución de producción de café en Nariño, y con el objetivo principal de conservar la biodiversidad de plantasyaves presentes en los paisajes cafeteros, 
se identificaron las especies de plantas usadas por las aves presentes en paisajes cafeteros en cuatro municipios del departamento de Nariño: Colón-Génova, La Unión, Arboleda y San Pedro de Cartago.

\section{MATERIALES Y MÉTODOS}

Área de estudio. Este trabajo se realizó en zonas rurales de cuatro municipios de tradición cafetera ubicados en el norte del departamento de
Nariño: Colón-Génova, La Unión, Arboleda y San Pedro de Cartago. En estas zonas fueron priorizadas un total de 28 herramientas de manejo del paisaje (HMP) en seis veredas de los cuatro municipios: Agroforestal=10, Bosque $=6$, Cafetal=4, Minicorredor $=4$, Cerca viva $=3$ y Árboles dispersos=1. En la Tabla 1 se describe la ubicación de cada vereda evaluada y en la Figura 1 la ubicación de las 28 herramientas de manejo del paisaje evaluadas.

Tabla 1. Ubicación de las veredas evaluadas en los cuatro municipios cafeteros del Norte de Nariño.

\begin{tabular}{|c|c|c|}
\hline Municipio & Vereda & Ubicación \\
\hline \multirow{2}{*}{ Colón-Génova } & La Victoria & $\begin{array}{l}\text { Entre los } 01^{\circ} 38^{\prime} 58.1^{\prime \prime} \mathrm{LN}-77^{\circ} 1^{\prime} 55.6^{\prime \prime} \mathrm{LO} \text { y } 01^{\circ} 39^{\prime} 16.4^{\prime \prime} \mathrm{LN}-77^{\circ} 2^{\prime} 7.7^{\prime \prime} \mathrm{LO} \text {, } \\
\text { entre } 1990 \text { - } 2268 \mathrm{msnm} \text {. Precipitación } 500 \text { y } 1000 \mathrm{~mm} \text {. Temperaturas: } \\
12 \text { a } 18^{\circ} \mathrm{C} \text { (Holdridge, } 1967 \text { ) (Figura } 1 \text { ). }\end{array}$ \\
\hline & $\begin{array}{l}\text { Bordo Alto-El } \\
\text { Púlpito }\end{array}$ & $\begin{array}{l}\text { Entre los } 01^{\circ} 38^{\prime} 45^{\prime \prime} \mathrm{LN}-77^{\circ} 0^{\prime} 4^{\prime \prime} \mathrm{LO} \text { y } 01^{\circ} 38^{\prime} 41^{\prime \prime} \mathrm{LN}-76^{\circ} 59^{\prime} 56.4^{\prime \prime L O} \text {, } \\
\text { entre } 2205 \text { - } 3219 \mathrm{msnm} \text {. Precipitación } 550 \text { y } 1000 \mathrm{~mm} \text {. Temperaturas: } \\
18 \text { a } 24^{\circ} \mathrm{C} \text { (Holdridge, } 1967 \text { ) (Figura } 1 \text { ). }\end{array}$ \\
\hline \multirow{2}{*}{ La Unión } & $\begin{array}{l}\text { Pradera A y } \\
\text { Pradera B }\end{array}$ & $\begin{array}{l}\text { Entre los } 01^{\circ} 35^{\prime} 48.2^{\prime \prime} \mathrm{LN}-77^{\circ} 9^{\prime} 6^{\prime \prime} \mathrm{LO} \text { y } 01^{\circ} 35^{\prime} 26.5^{\prime \prime} \mathrm{LN}-77^{\circ} 8^{\prime} 58.7^{\prime \prime} \mathrm{LO} \text {, } \\
\text { entre } 1840-1937 \mathrm{msnm} \text {. Precipitación } 1000 \text { a } 2000 \mathrm{~mm} \text {. Temperatura } \\
\text { mayor a } 12^{\circ} \mathrm{C} \text { (Holdridge, 1967) (Figura } 1 \text { ). }\end{array}$ \\
\hline & Las Palmas & $\begin{array}{l}\text { Entre los } 01^{\circ} 35^{\prime} 48.2^{\prime \prime} \mathrm{LN}-077^{\circ} 9^{\prime} 6^{\prime \prime} \mathrm{L} 0 \text { y } 01^{\circ} 35^{\prime} 26.5^{\prime \prime} \mathrm{LN}-77^{\circ} 8^{\prime} 58.7^{\prime \prime} \mathrm{LO} \text {, } \\
\text { entre } 2476 \text { - } 2736 \mathrm{msnm} \text {. Precipitación } 500 \text { y } 1000 \mathrm{~mm} \text {. Temperatura } \\
\text { de } 6-12^{\circ} \mathrm{C} \text {. (Holdridge, 1967) (Figura 1). }\end{array}$ \\
\hline Arboleda & Santa Teresa & $\begin{array}{l}\text { Entre los } 1^{\circ} 28^{\prime} 39.4^{\prime \prime} \mathrm{LN}-77^{\circ} 7^{\prime} 42.8^{\prime \prime} \mathrm{LO} \text { y } 1^{\circ} 28^{\prime} 47.6^{\prime \prime} \mathrm{LN}-7^{\circ} 7^{\prime} 42.3^{\prime \prime} \mathrm{LO} \text {, } \\
\text { entre } 1900-2100 \mathrm{msnm} \text {. Precipitación } 1000 \text { y } 2000 \mathrm{~mm} \text {. Temperaturas } \\
\text { mayores a los } 12^{\circ} \mathrm{C} \text { (Holdridge, 1967) (Figura } 1 \text { ). }\end{array}$ \\
\hline \multirow[t]{2}{*}{ San Pedro de Cartago } & El Salado & $\begin{array}{l}\text { Entre los } 1^{\circ} 34^{\prime} 46.2^{\prime \prime} \mathrm{LN}-77^{\circ} 6^{\prime} 46.9^{\prime \prime} \mathrm{LO} \text { y } 1^{\circ} 33^{\prime} 56.6^{\prime \prime} \mathrm{LN}-77^{\circ} 6^{\prime} 29.1^{\prime \prime} \mathrm{LO} \text {, } \\
\text { entre } 1900 \text { - } 2142 \mathrm{msnm} \text {. Presenta una temperatura media de } 18^{\circ} \mathrm{C} \text { y } \\
\text { precipitación media anual de } 1.600 \mathrm{~mm} \text {. (Figura } 1 \text { ). }\end{array}$ \\
\hline & La Chorrera & $\begin{array}{l}\text { Ubicada entre los } 1^{\circ} 34^{\prime} 18^{\prime \prime} \mathrm{LN}-77^{\circ} 6^{\prime} 46.1^{\prime \prime} \mathrm{LO} \text { y } 1^{\circ} 34^{\prime} 18.3^{\prime \prime} \mathrm{LN}- \\
77^{\circ} 6^{\prime} 40.9^{\prime \prime} \mathrm{L} 0 \text {, entre } 1900-2100 \mathrm{msnm} \text { (Figura 1). }\end{array}$ \\
\hline
\end{tabular}




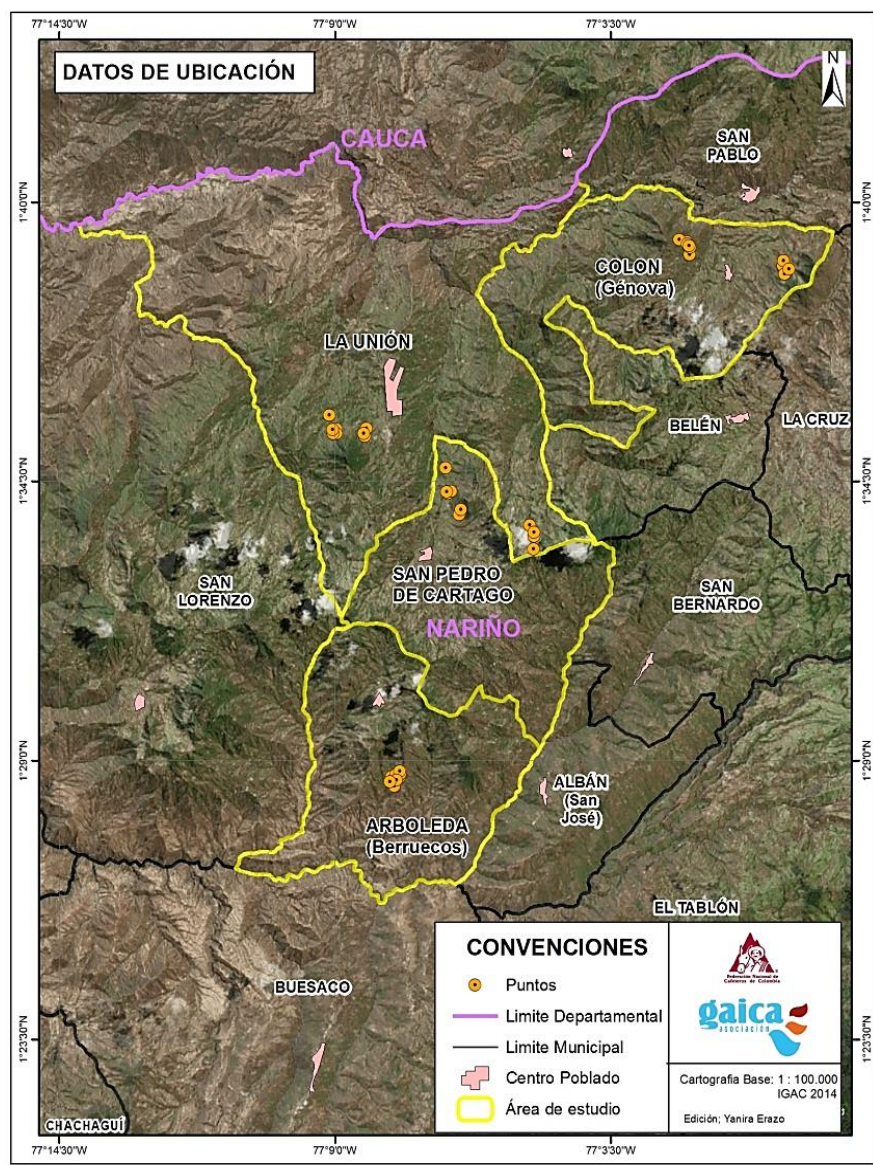

Figura 1. Ubicación de las 28 unidades del paisaje evaluadas en los cuatro municipios cafeteros del Norte de Nariño.

En la Tabla 2 se describen las unidades del paisaje evaluadas en los cuatro municipios cafeteros del Norte de Nariño.

Tabla 2. Ubicación de las unidades de paisaje evaluadas en los cuatro municipios cafeteros del Norte de Nariño.

\begin{tabular}{|c|c|c|}
\hline Municipio & Vereda & Herramienta de paisaje evaluada \\
\hline \multirow{7}{*}{ Colón - Génova } & \multirow{5}{*}{ La Victoria } & Bosque seco montano bajo (bs-MB). \\
\hline & & Cafetal con sombra. \\
\hline & & Sistema Agroforestal. \\
\hline & & Cerca viva. \\
\hline & & Minicorredor. \\
\hline & \multirow{2}{*}{ Bordo Alto - El Pulpito. } & Bosque seco premontano (bs-PM). \\
\hline & & Minicorredor. \\
\hline
\end{tabular}




\begin{tabular}{|c|c|c|}
\hline Municipio & Vereda & Herramienta de paisaje evaluada \\
\hline \multirow{7}{*}{ La Unión. } & \multirow{5}{*}{ Pradera A y Pradera B. } & Bosque húmedo montano bajo (bh-MB). \\
\hline & & Cafetal con sombra. \\
\hline & & Sistema Agroforestal. \\
\hline & & Minicorredor. \\
\hline & & Cerca Viva. \\
\hline & \multirow{2}{*}{ Las Palmas. } & Bosque húmedo montano (bh-M). \\
\hline & & Minicorredor. \\
\hline \multirow{3}{*}{ Arboleda } & \multirow{3}{*}{ Santa Teresa. } & Bosque. \\
\hline & & Cafetal con sombra. \\
\hline & & Sistema Agroforestal. \\
\hline \multirow{4}{*}{$\begin{array}{l}\text { San Pedro de } \\
\text { Cartago }\end{array}$} & \multirow{2}{*}{ El Salado. } & Bosque. \\
\hline & & Árboles dispersos. \\
\hline & \multirow{2}{*}{ La Chorrera } & Cafetal con sombra. \\
\hline & & Sistema Agroforestal. \\
\hline
\end{tabular}

Trabajo en campo. La investigación se realizó de septiembre a diciembre de 2012. Para determinar las especies de plantas usadas por las aves presentes en paisajes cafeteros se realizaron observaciones en campo de especies vegetales a partir de recorridos a lo largo de cada unidad de paisaje (bosque, cafetal con sombra, agroforestal, minicorredor, árboles dispersos y cerca viva). En cada caso, se tomaron datos de coordenadas geográficas, fecha, zona, área evaluada, altitud, nombre común y cuando fue posible, la identificación taxonómica de las especies vegetales en campo, estimación de la altura total de la planta (en metros), hábito o estratificación. Se realizaron colectas generales de material vegetal a través del método de herborización para realizar la determinación taxonómica de las especies vegetales. Se hizo énfasis en la colecta de especies vegetales que fueron visitadas por especies de aves de la zona, bien sea para percha, consumo o nidificación. El material colectado en campo después de su herborización se identificó en el herbario PSO de la Universidad de Nariño.
Para la consideración de los taxones se siguió el sistema de clasificación APG III-The Angiosperm Phylogeny Group (2009) y se revisó el árbol filogenético propuesto por Cole y Hilger (2010). Para la correcta escritura de los nombres científicos y sus autores, se consultó la base de datos Tropicos del Missouri Botanical Garden (2012). Se determinaron especies de plantas como fuente potencial de recursos para las aves en las áreas muestreadas de la zona cafetera del departamento de Nariño.

\section{RESULTADOS Y DISCUSIÓN}

El análisis de los paisajes cafeteros en las áreas de estudio permitieron determinar que se trata de hábitats altamente intervenidos, a excepción del bosque de la vereda Las palmas (municipio de La Unión) con vegetación característica de bosque maduro, mayor cobertura arbórea y mayores alturas de dosel con predominancia de especies de las familias Cunoniaceae de 15 - 20m de altura, Primulaceae, Euphorbiaceae, Pentaphylacaceae, Chloranthaceae, Adoxaceae, 
Clusiaceae, Rubiaceae con individuos desde 3 $12 \mathrm{~m}$ de altura.

Entre las herramientas de manejo implementadas se destaca el minicorredor de la vereda Bordo Alto (municipio de Colón-Génova), que ocupa un área de aproximadamente 16ha y permite la conexión de bosques hasta unirse con el cerro El Púlpito, presenta especies que han sido sembradas hace varios años como Arrayán (Myrcia popayanensis Hieron.), Guayacán (Lafoensia acuminata Ruíz \& Pav.), Encino (Weinmannia pubescens Kunth.), Clethra fagifolia Kunth, Aguacatillo (Persea caerulea (Ruiz \& Pav.) Mez.), que alcanzan alturas de hasta $8 \mathrm{~m}$. El cafetal con sombra de la vereda Santa Teresa (municipio de Arboleda), que presenta una alta cobertura arbórea, con doseles que alcanzan de 6 a 10m de altura, favoreciendo los sitios de percha y una mayor oferta de recursos alimenticios para las aves de la zona. Las especies que brindan sombrío fueron sembradas desde hace 10 - 20 años y pertenecen a las familias Myrtaceae, Fabaceae, Lauraceae, Rutaceae, Solanaceae. Algunos sistemas agroforestales de la vereda Pradera A (municipio de La Unión), que se encuentran mejor implementados y más tecnificados, la vegetación asociada está compuesta por especies frutales, que están dispersas a lo largo del cultivo y presentaron alta cobertura arbórea.

Se encontraron 25 especies vegetales que son consumidas por aves frugívoras, entre ellas se destacan Moquillo (Saurauia brachybotrys Turcz, Saurauia pruinosa R.E. Schultes), Pelotillo (Viburnum triphyllum Benth.), Charmolán (Geissanthus sp.), Cucharo (Myrsine coriacea (Sw.) R. Br. ex Roem. \& Schult.y Myrsine guianensis (Aubl.) Kuntze), Zurrumbo (Trema micrantha (L.) Blume), Poroto (Erythrina edulis Triana ex Micheli), Frutillo (Senna pistaciifolia (Kunth) H.S. Irwin \& Barneby), Vainillo (Senna spectabilis (DC.) H.S. Irwin \& Barneby), Yarumo
(Cecropia sp), ímpamo (Clusia ellipticifolia Cuatrec.), Ímpamo mambito (Clusia sp.), Arnalulo (Cavendishia bracteata (Ruíz \& Pav. ex J. St.-Hil.) Hoerold), Guásimo (Cordia acuta Pittier), Guamo (Inga densiflora Benth., Inga edulis Mart.), Guayacán (Lafoensia acuminata (Ruíz \& Pav.) DC.), Arrayán (Myrcia popayanensis Hieron.), Níspero (Eriobotrya japónica (Thunb.) Lindl.), Cascarillo (Cinchona pubescens Vahl), Laurel (Morella pubescens (Humb. \& Bonpl. ex Willd.) Wilbur), Phoradenderon sp., que se constituyen en la principal dieta para aves como Vireo olivaceus, Tangara xanthocephala, Tangara vitriolina, Drendoica fusca, Saltator atripennis, Piranga flava, Euphonia cyanocephala, Myioborus miniatus, entre otras (Tabla 3).

Ocho especies vegetales: Nacedero (Trichanthera gigantea (Bonpl.) Nees), Cajeto (Delostoma integrifolium D. Don.), Poroto (Eritryna edulis Triana ex Micheli), Guayacán (Lafoensia acuminata (Ruiz \& Pav.) DC.), Quillotocto (Tecoma stans (L.) Juss. ex Kunth), Arnalulo (C. bracteata), Guamo (I. densiflora), Iochroma fuchsioides (Bonpl.) Miers, son aprovechadas por aves nectarívoras, específicamente por Colibríes y Robamieles como Amazilia saucerrottei, Aglaiocercus kingi, Coeligena coeligena, Adelomyia melanogenys, Ocreatus underwoodii, Amazilia franciae, Diglossa sittoides. El Aliso (Alnus acuminta Kunth) es aprovechado por algunas aves semilleras como Pheucticus aureoventris, Sporophila luctuosa, Atlapetes albinucha, Atlapetes latinuchus, Atlapetes schistaceus, Astragalinus psaltria (Tabla 3). 


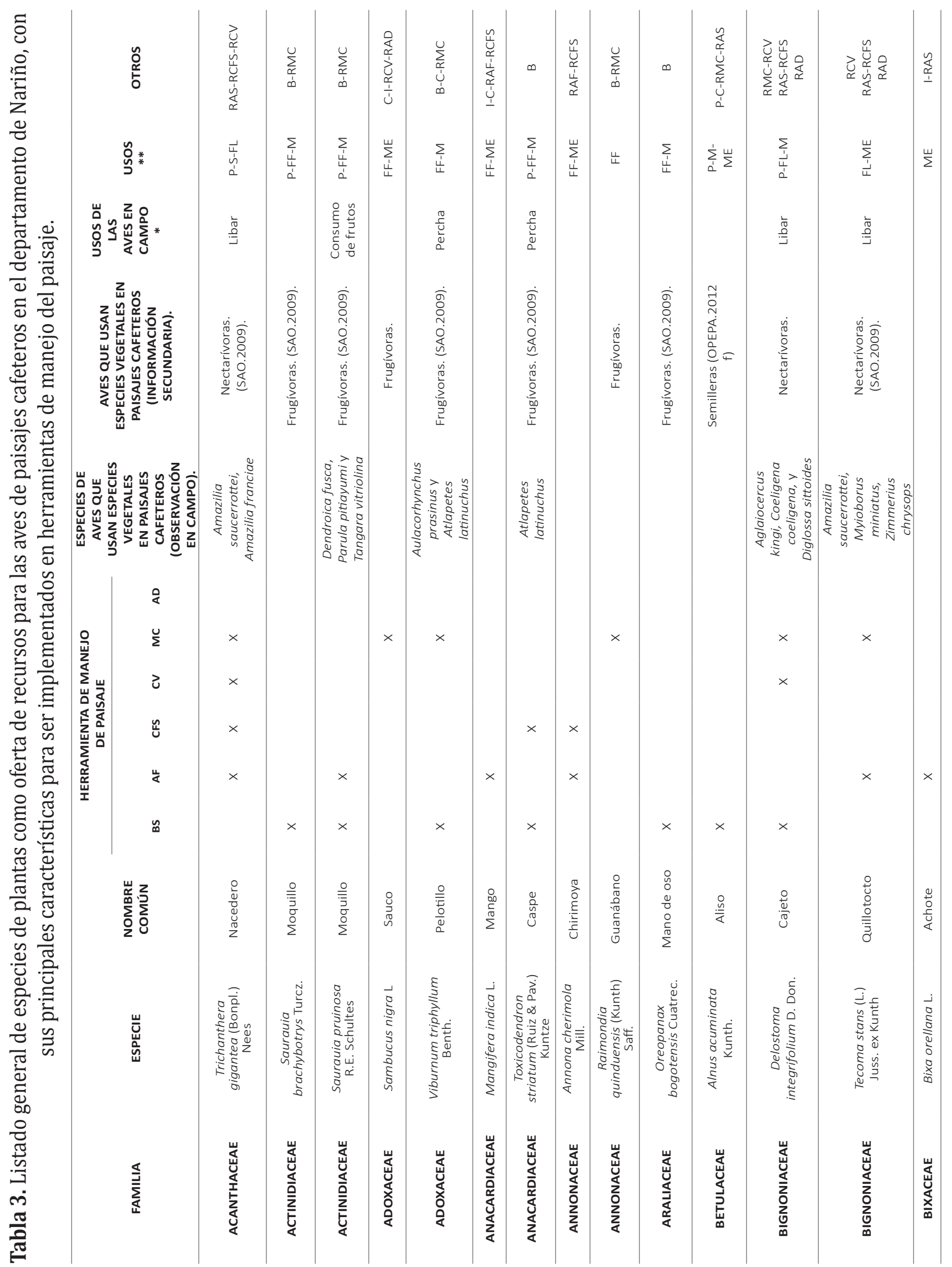




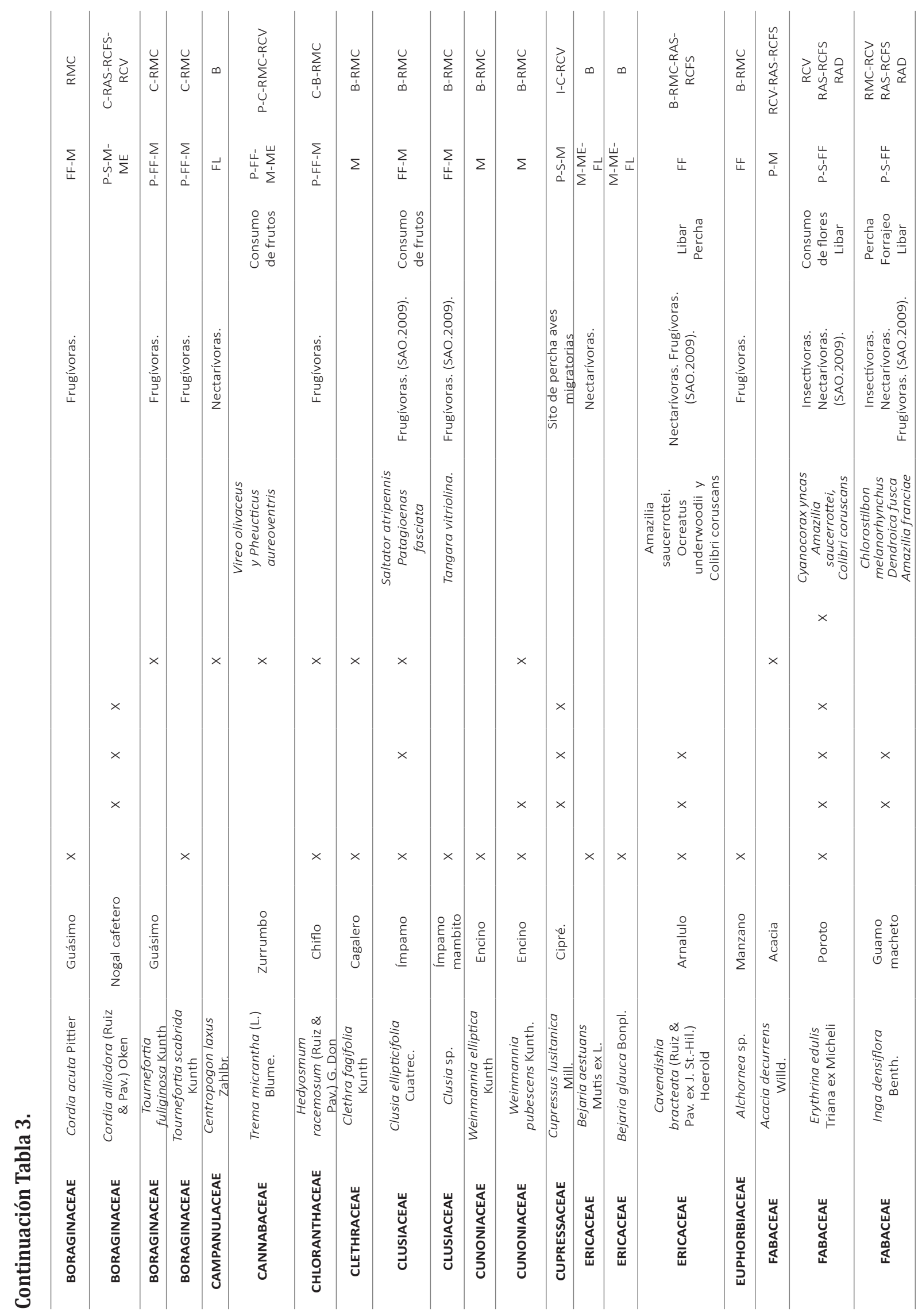




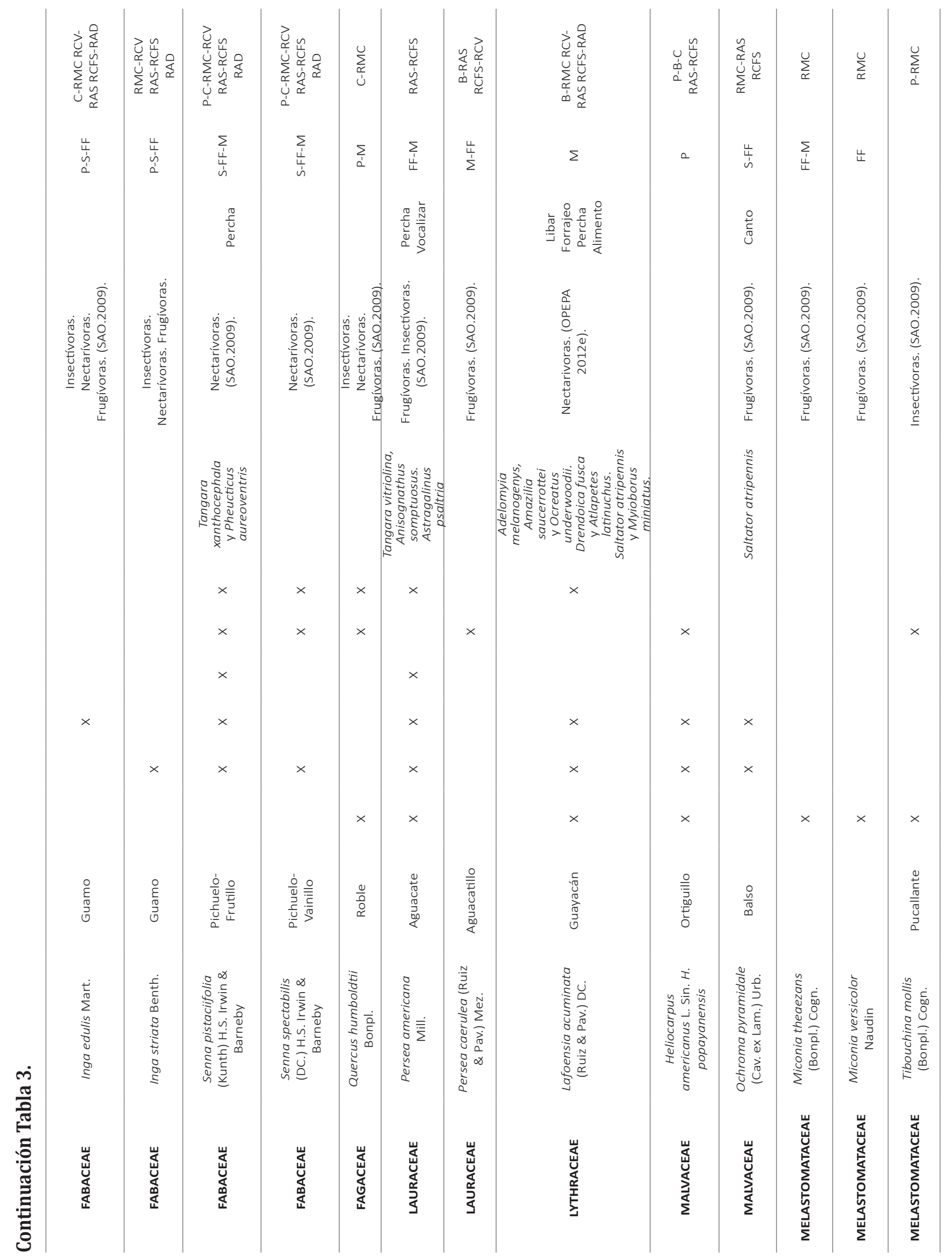




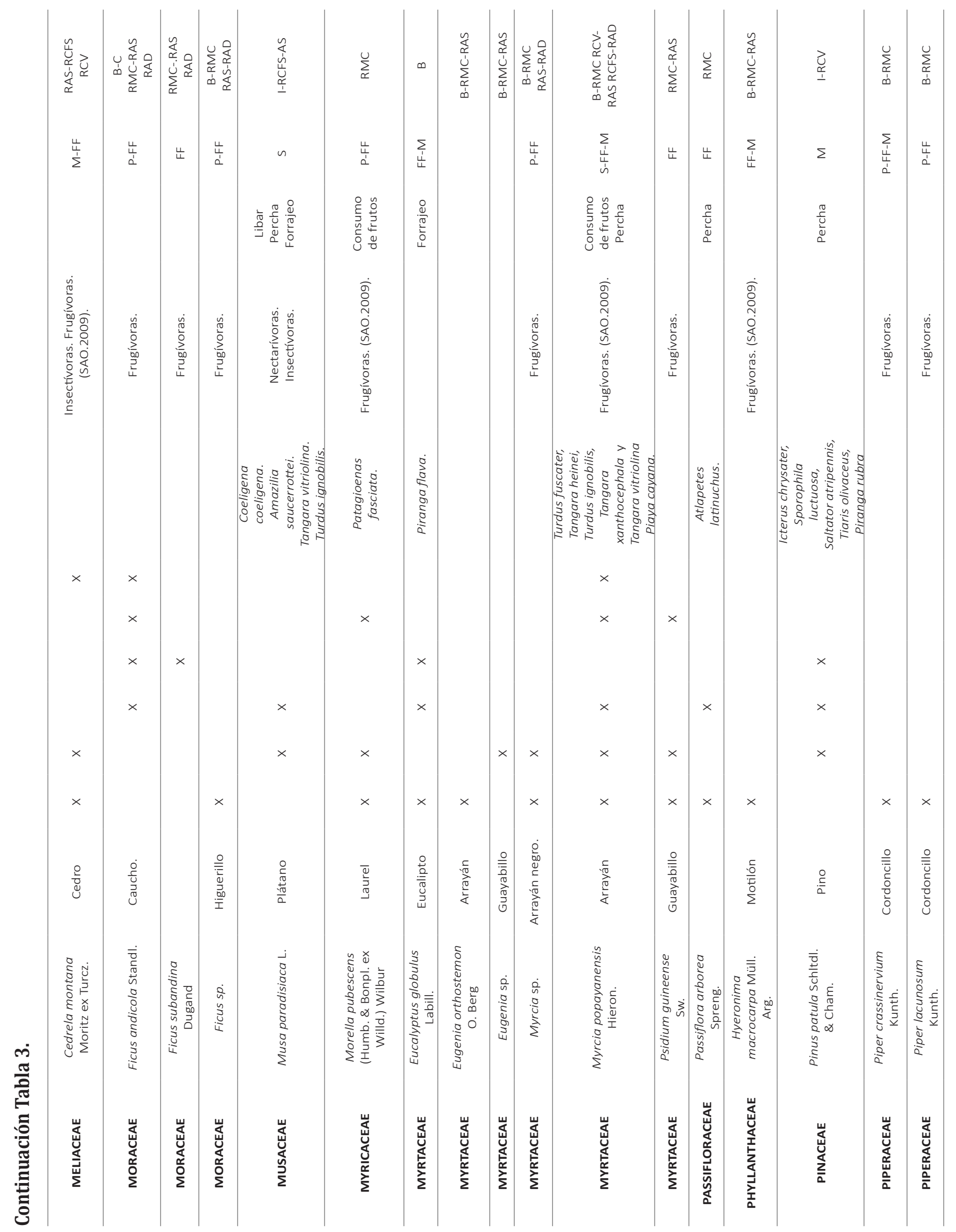




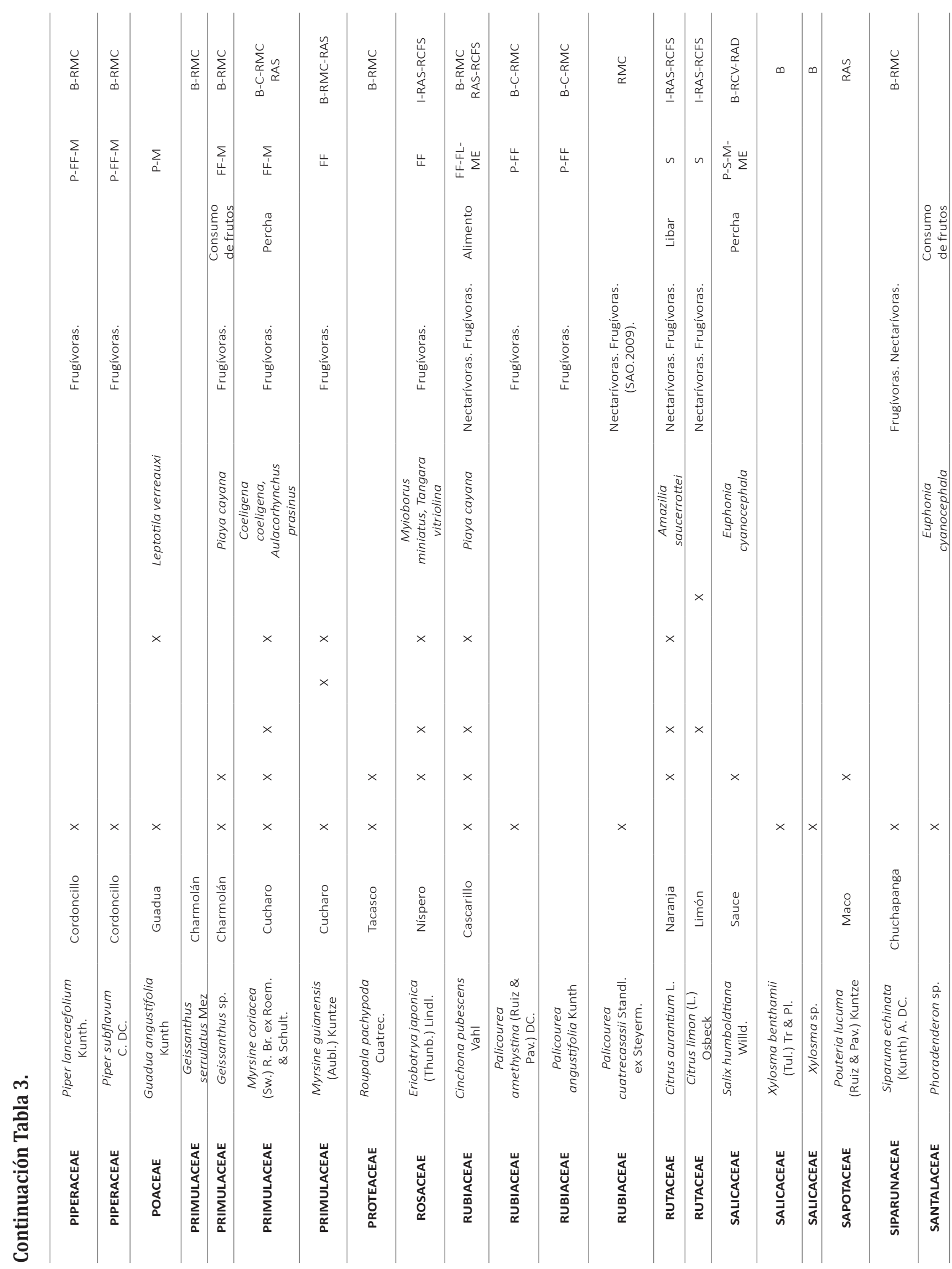




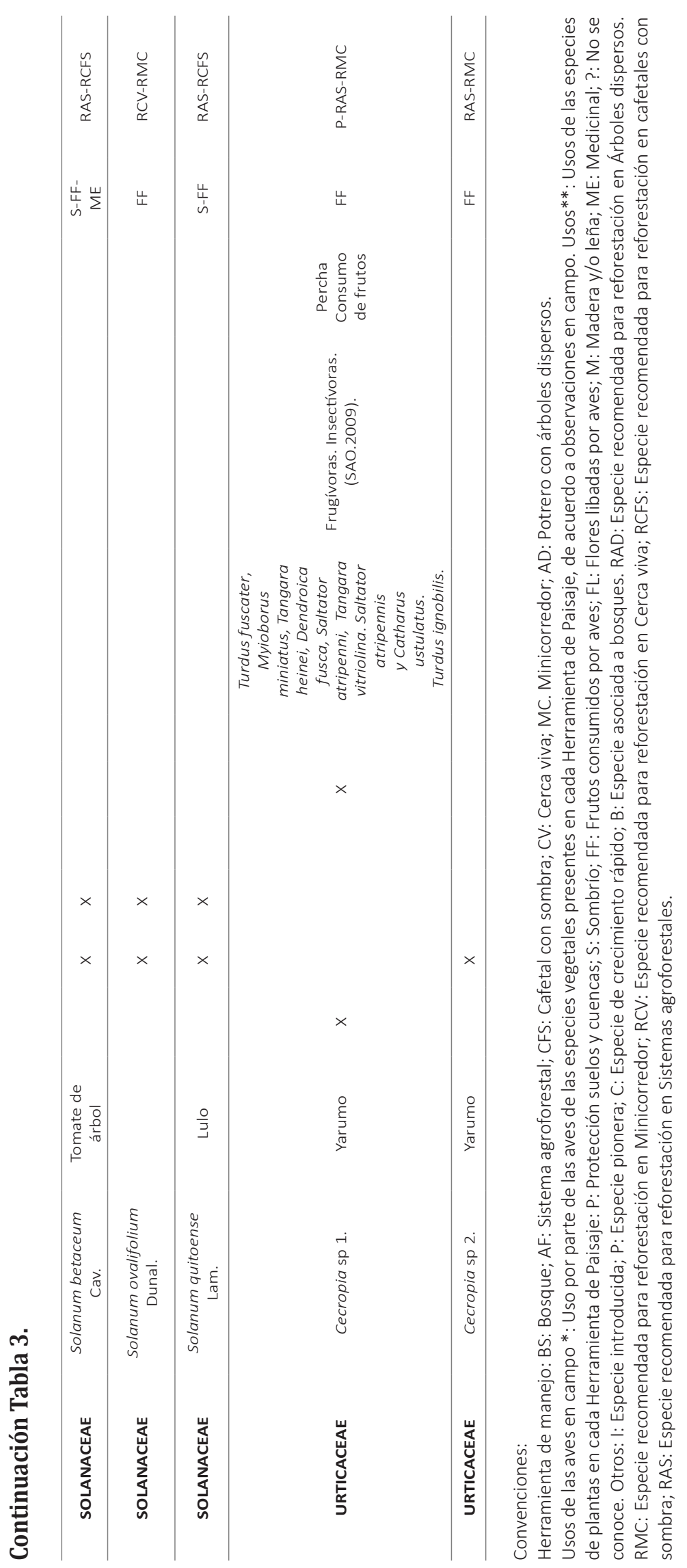

UNIVERSIDAD DE NARIÑO 
Nueve especies vegetales como Pucallante ( Tibouchina mollis (Bonpl.) Cogn.), Cedro (Cedrela montana Moritz ex Turcz.), Achiote (Bixa OreIlana L.), yarumos (género Cecropia), Guamo (I. densiflora, I. edulis), poroto (E. edulis) y Roble (Q. humboldtii) son aprovechadas por aves que incluyen insectos en su dieta, como: Contopus sordidulus, Tyrannus melancholicus, Contopus cooperi, Phyllomyias uropygialis, Phyllomyias nigrocapillus, Elaenia pallatangae (Tabla 3).

De acuerdo con información secundaria, de las especies de plantas registradas en los municipios evaluados, por lo menos 40 especies podrían proporcionar recursos para las aves frugívoras, doce para las aves nectarívoras y nueve para las aves insectívoras, destacando que dichos recursos pueden aumentar si se promueve la recuperación e incremento de la cobertura vegetal en cada una de las áreas evaluadas. Especies como Arrayán (M. popayanensis), Cucharo (M. coriacea, M. guianensis), Charmolán (Geissanthus sp), Guamos (I. densiflora, I. edulis), Guayacán ( $L$. acuminata), Nacedero (T. gigantea), Quillotocto (T. stans) y Cajeto (D. integrifolium) están entre las más frecuentadas por las aves de las zonas, lo cual se explica debido al tipo de frutos que estas especies poseen como es el caso de las bayas carnosas de $M$. popayanensis que son apetecidas por aves frugívoras, como Pavas, Tucanes y Saltarines. Los frutos pequeños de M. coriacea y M. guianensis, son consumidos por Mirlas, Elaenias, Cotingas y Clarineros, los cuales dispersan las semillas junto con sus excrementos (OPEPA, 2012a). Frutos de V. tryphyllum y de M. pubescens son consumidos por Carpinteros, Mirlas, Cotingas y por Tucanes como Aulacorhynchus prasinus (OPEPA, 2012b, c).

Las llamativas flores de ciertas especies también se constituyen en atractivo para las aves nectarívoras, como las flores de coloración blanca y de gran tamaño de L. acuminata, las abundantes flores tubulares con olor dulce de $D$. integrifolium, las flores amarillas y vistosas de $T$. stans, que suelen ser frecuentadas por especies de colibríes como Colibri coruscans (OPEPA, 2012d) y abundantes flores de coloración roja de I. fuchsioides considerada una especie importante como melífera por el abundante néctar que ofrece a las aves especialmente a Colibríes como 0 . underwoodii.

\section{Especies vegetales en procesos de restaura-} ción. Según Beer et al. (2004) es necesario, en los procesos de restauración, aprovechar los beneficios ambientales que ciertas especies vegetales pueden ofrecer, como producción (madera, leña, forraje, frutas, medicinas, entre otros) y servicios (sombra para cultivos y/o animales, protección como en el caso de cercas vivas, entre otros). En este sentido se propone un listado de especies, algunas nativas y otras introducidas, con sus principales características (Tabla 3 ), las cuales pueden ser utilizadas en los diferentes procesos de manejo de estos paisajes, a través de la implementación de estrategias de producción y propagación que permitan a su vez conservar y contrarrestar la pérdida de biodiversidad tanto de flora como de fauna asociada a este tipo de paisajes.

En minicorredores. Debido a que las especies nativas de cada región ya están adaptadas a las condiciones de la localidad donde se encuentran, incorporarlas dentro o alrededor de los sistemas productivos permite conservar más biodiversidad que si se utilizan especies introducidas. Se recomienda por tanto, la implementación de especies asociadas a bosque, logrando la conexión entre parches o fragmentos que se encuentren en las zonas (Tabla 3).

En sistemas agroforestales. La elección de un sistema con árboles para sombra depende de la necesidad de diversificar la producción (Jiménez 
y Muschler, 2001). Se recomienda realizar una combinación simultánea de algunos árboles que se especifican en la Tabla 3 con los diferentes cultivos de café, y así contribuir al mejoramiento de las condiciones del suelo, aportar recursos maderables y productos adicionales de consumo como frutales, en asociaciones con especies fijadoras de nitrógeno, principalmente de la Familia Fabaceae como Guamos, Frutillo, Vainillo y lo más importante incrementar la diversidad de avifauna asociada a esta herramienta de paisaje. La combinación de especies puede darse entre fijadoras de nitrógeno, especies maderables como C. alliodora, C. odorata, $C$. montana o especies frutales como Tomate de árbol (S. betaceum), Lulo (S. quitoense), Mango (M. indica), Aguacate (P. americana), Aguacatillo (P. caerulea), Níspero (E. japonica), Naranja (C. aurantium), Limón (C. limón) y Plátano (M. paradisiaca).

En cafetales con sombra. Los cafetales con sombra brindan muchos bienes y servicios a la sociedad. Al ser hábitat de animales y plantas silvestres, ayudan a mantener la biodiversidad. Los árboles usados como sombrío son importantes productores de oxígeno y contribuyen a disminuir el calentamiento del planeta (Muschler, 1999). Igualmente, se recomiendan especies de la familia Fabaceae, además de otras especies, que aunque no se reportaron en las zonas pueden servir como sombrío, tales como: Leucaena leucocephala (Lam.) de Wit (Leucaena), Gliricidia sepium Kunth ex Steud. (Matarratón), Tabebuia rosea (Bertol.) DC. (Apamate), en asociación con plátano y maíz; Bambusa guadua Bonpl. (Bambú) por su gran flexibilidad y resistencia, es recomendada para cercas vivas, sombrío de café y es fertilizante de suelos; Montanoa quadrangularis Sch. Bip. (Arboloco) puede ser usada para prevenir la erosión, proteger fuentes hídricas y atraer entomofauna (insectos).
Cercas vivas. El establecimiento de cercas vivas bien implementadas y con adecuado manejo permite una reducción en costos, en la presión sobre el bosque por la búsqueda de postes y leña, y lo más importante ofrece follaje en cantidad y de calidad durante la época seca, permitiendo ofrecer recursos para la comunidad y para las aves asociadas a este tipo de paisaje.

Beer et al. (2004) sugieren el uso de árboles y arbustos, formando hileras con el fin de mejorar el suelo a partir de la fijación de nitrógeno y/o reducir erosión en pendientes. Para lo anterior, se recomienda sembrar leñosas para la delimitación de potreros, casi siempre complementada con el uso de alambre de púas. Las cercas vivas con adecuado manejo son útiles para reemplazar las cercas de alambre, duran más tiempo y disminuyen los costos (Jiménez y Muschler, 2001).

Potreros con árboles dispersos. La implementación de este sistema tiene como objetivo principal mejorar la actividad ganadera y lograr de manera secundaria la producción de madera, leña o frutos. Se recomienda sembrar especies de más de $20 \mathrm{~cm}$ de diámetro como Poroto (E. edulis), Cedro (C. montana), Nacedero (T. gigantea), Quillotocto (T. stans), Cajeto (D. integrifolium), Caucho (F. andicola), Nogal cafetero (C. alliodora), Sauco (S. nigra) y Acacia (A. decurrens). Estas dos últimas que por sus condiciones de rápido crecimiento y facilidad de rebrote, permiten el consumo directo de forraje de alto valor nutricional para los animales mediante el ramoneo de los árboles. En la Tabla 3 se describen las especies que pueden ser utilizadas en esta herramienta, caracterizada por la sombra y refugio que ofrece a la fauna de la zona y por los bienes y servicios en términos de producción de leña y frutales. 


\section{CONCLUSIONES}

La oferta de frutos y flores disponibles en la zona cafetera de Nariño está representada por las familias Primulaceae, Clusiaceae, Chloranthaceae, Fabaceae, Lythraceae, Myrtaceae, Solanaceae, Melastomataceae y Bignoniaceae que se caracterizan por presentar frutos con semillas que pueden ser explotadas por especies de la familia Thraupidae. Igualmente se destacan especies de la familia Moraceae, Rubiaceae y Adoxaceae que son fuente importante de alimento para las aves especialistas como Tucanes que son altamente dependientes de este alimento.

Especies de familias nativas como Primulaceae, Clusiaceae, Chloranthaceae, Myrtaceae, Melastomataceae, Ericaceae, Lythraceae, Rubiaceae, especies de familias introducidas y especies de familias frutales que ofrecen frutos, flores o semillas como fuente de recursos para las aves, son recomendadas para ser implementadas en las diferentes herramientas de paisaje con el fin de promover la recuperación e incremento de la cobertura vegetal tanto de los ecosistemas boscosos como en cada una de las unidades de paisaje evaluadas.

\section{AGRADECIMIENTOS}

Los resultados presentados son producto del esfuerzo colectivo de los integrantes del equipo de investigación de la Asociación GAICA, quienes ejecutaron el proyecto denominado "Incorporación de la biodiversidad en el sector cafetero en Colombia. Aves del paisaje cafetero en el departamento de Nariño, municipios Colón-Génova, La Unión, Arboleda y San Pedro de Cartago", apoyado y financiado por la Federación Nacional de Cafeteros de Colombia. Agradecemos a la coordinación departamental, a los guías de campo, a las familias caficulturas, a la geógrafa Yanira Erazo por la edición de la cartografía base, a los ornitólogos Ronald Armando Fernández y Fiorela Delgado Chaves, al investigador Pedro José Cardona Camacho por su colaboración y todo el aprendizaje compartido durante las largas jornadas de campo en los hermosos paisajes cafeteros de Nariño.

\section{REFERENCIAS BIBLIOGRÁFICAS}

1. APG III - The Angiosperm Phylogeny Group. 2009. An update of the Angiosperm Phylogeny Group classification for the orders and families of flowering plants: APG III. Botanical Journal of the Linnean Society. 161:105 - 121. doi: 10.1111/j.1095-8339.2009.00996.x.

2. Asociación Gaica - Federación Nacional de Cafeteros. 2012. Aves Del Paisaje Cafetero en el Departamento de Nariño Municipios Colón-Génova, La Unión, Arboleda y San Pedro De Cartago. Proyecto Incorporación de la Biodiversidad En El Sector Cafetero En Colombia. Asociación Gaica, San Juan de Pasto, Colombia. 146p.

3. Beer, J.; Ibrahim, M.; Somarriba, E.; Barrance, A.; Leakey R. 2004. Establecimiento y manejo de árboles en sistemas agroforestales. Capítulo 6. Árboles de Centroamérica. OFICATIE. 46p.

4. Borkhataria, R.; Collazo, J. A.; Groom, M. J.; Jordan-Garcia, A. 2012. Shade-Grown coffee in Puerto Rico: Opportunities to preserve biodiversity while reinvigorating a struggling agricultural commodity. Agriculture, Ecosystem and Enviroment. 149:164 - 170. doi: 10.1016/j. agee.2010.12.023.

5. Borrero, J. I. 1986. "La substitución de cafetales de sombrío por caturrales y su efecto negativo sobre la fauna". Caldasia. 15:725 - 732. doi: 10.15446/caldasia.

6. Cole, T.; Hilger, H. 2010. Poster "Angiosperm phylogeny-APG III. En: http://www2.biologie. fu-berlin.de/sysbot/poster/poster1.pdf.1p; consulta: diciembre, 2012. 
7. Giraldo, P.; Laina, A.; Lopez, J. H.; Aguirre, J.F.; Toro, Y. 2009. Café, una alternativa para la conservación. Wildlife Conservation Society, WWF Colombia, Asociación Red Colombiana de Reservas Naturales de la Sociedad Civil y Federación Nacional de Cafeteros. Primera edición, Santiago de Cali, Colombia. 76p.

8. Holdridge, L. R. 1967. Life Zone Ecology. Tropical Science Center. San José, Costa Rica. 206p.

9. Jiménez, F.; Muschler, R. 2001. Introducción a la agroforestería. Funciones y aplicaciones de sistemas agroforestales. Módulos de Enseñanza Agroforestal. CATIE/GTZ. 24p.

10. Missouri Botanical Garden's Vast (Vascular Trópicos). 2012. Nomenclatural Database and Associated Authority Files. En: http://www. tropicos.org. 1 p; consulta: diciembre, 2012.

11. Muschler, R. G. 1999. Árboles en cafetales.. Proyecto Agroforestal CATIE/GTZ. Modulo No. 5. CATIE. Turrialba, Costa Rica. 139p.

12. OPEPA - Organización para la Educación y Protección Ambiental. 2012a. Cucharo Colorado, Myrsine guianensis. En: http://www.opepa. org/index.php?option=com_content\&task=vie w\&id=327\&Itemid=30>. 1 p.; consulta: diciembre, 2012.

13. OPEPA. Organización para la Educación y Protección Ambiental. 2012b. Chuque, Viburnum triphyllum. En: http://www.opepa.org/index. php?option=com_content $\&$ task=view\&id=349 \&Itemid=30). 1 p.; consulta: diciembre, 2012.

14. OPEPA. Organización para la Educación y Protección Ambiental. 2012c. Laurel de Cera, Morella pubescens. En: http://www.opepa.org/

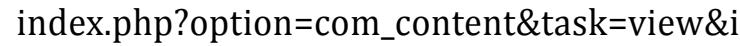
d=557\&Itemid=30). 1 p.; consulta: diciembre, 2012.

15. OPEPA. Organización para la Educación y Protección Ambiental. 2012d. Chicalá, Tecoma stans. En: https://sites.google.com/a/nogales. edu.co/plants-in-colegio-los-nogales/home/ chical. 1 p.; consulta: diciembre, 2012.
16. OPEPA. Organización para la Educación y Protección Ambiental. 2012e. Guayacán de Manizales, Lafoensia acuminata. En: https://sites. google.com/a/nogales.edu.co/plants-in-colegio-los-nogales/home/guayacn-de-manizales. 1 p.; consulta: diciembre, 2012.

17. OPEPA. Organización para la Educación y Protección Ambiental. 2012 f. Aliso, Alnus acuminata. En: https://sites.google.com/a/nogales. edu.co/plants-in-colegio-los-nogales/aliso. 1 p.; consulta: diciembre, 2012.

18. Perfecto, I.; Rice, R.A.; Greenberg, R.; Van Der Voot. M. E. 1996. Shade Coffee: A disappearing refuge for biodiversity. Bioscience. 46(8):598 $-608$.

19. Rappole, J. H.; King, D. I.; Vega Rivera. J. H. 2003. Coffee and Conservation. Conservation Biology. 17(1):334 - 336.

20. SAO - Sociedad Antioqueña de Ornitología. 2009. Vida, Color y Canto. Plantas neotropicales que atraen aves. Mesaeditores, Medellín. 288p. 